\title{
Transmissão da Política Monetária Pelos Canais de Tomada de Risco e de Crédito: Uma Análise Considerando os Seguros Contratados pelos Bancos e o Spread de Crédito no Brasil
}

\author{
Debora Pereira Tavares*, Gabriel Caldas Montes ${ }^{\dagger}$, Osmani Teixeira de \\ Carvalho Guillén ${ }^{\ddagger}$
}

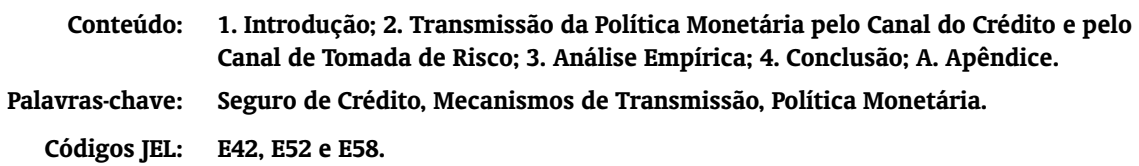

Este trabalho apresenta uma contribuição pioneira para a literatura sobre mecanismos de transmissão da política monetária pelos canais do crédito e de tomada de risco ao analisar a influência da política monetária sobre o processo de contratação de seguro referente às perdas com operações de concessão de crédito bancário às pessoas físicas e investigar o impacto exercido pela contratação desta modalidade de seguro sobre o spread de crédito no Brasil. Os resultados indicam que: i) políticas monetárias influenciam o prêmio do seguro de crédito; e ii) existe uma relação positiva entre o prêmio de seguro e o spread, sugerindo que o spread de crédito é sensível ao montante de seguros pagos pelas entidades financeiras.

This research presents a pioneering contribution to the literature on the transmission mechanism of monetary policy through the credit channel and the risk-taking channel, since it analyzes the influence of monetary policies on the insurance hiring process by banks in order to protect them against losses in lending transactions to individuals and, also, investigates the impact of contracting this type of insurance on credit spread in Brazil. The results indicate that: i) monetary policies influence the credit insurance

\footnotetext{
*IRB-Brasil Resseguros S.A. e IBMEC. E-mail: debuf $r j @ y$ ahoo.com.br

${ }^{\dagger}$ Universidade Federal Fluminense, Departamento de Economia; Conselho Nacional de Pesquisa (CNPq). E-mail: gabrielmontesuff@yahoo.com.br

${ }^{\ddagger}$ Banco Central do Brasil e IBMEC. E-mail: osmani . guillen@bcb.gov . br
} 
premium; and ii) there exists a positive relationship between the insurance premium and the spread, suggesting that the credit spread is sensitive to the amount of insurance paid by financial institutions.

\section{INTRODUÇÃO}

A literatura tradicional relacionada aos mecanismos de transmissão da política monetária geralmente enfatiza cinco canais de transmissão: taxa de juros, taxa de câmbio, preços de ativos, crédito e expectativa (Mishkin, 1995, Montes, 2013). Embora uma análise acerca de todos esses canais de transmissão seja relevante, episódios recentes (por exemplo, como o da crise do subprime) aumentaram a necessidade de estudar o canal de crédito em particular. ${ }^{1}$

Por sua vez, outro tipo de mecanismo de transmissão da política monetária vem ganhando grande atenção, e foi recentemente denominado como canal de tomada de risco (risk-taking channel). Este canal diz respeito a como as mudanças na taxa de juros de política monetária afetam tanto a percepção de risco como também a tolerância ao risco dos agentes. Contudo, poucos estudos foram elaborados acerca da ligação entre a política monetária e a percepção de risco e decisões dos agentes econômicos, ou seja, pouca atenção tem sido dada ao canal de tomada de risco (Borio e Zhu, 2008).

Este trabalho analisa a influência das diferentes formas de política monetária sobre o processo de contratação de seguro referente às perdas com operações de concessão de crédito bancário às pessoas físicas (Seguro de Crédito - Pessoa Física) e investiga o impacto exercido pela contratação desta modalidade de seguro sobre o spread de crédito no Brasil, e, portanto, sobre o risco assumido por bancos com essas operações.

A literatura relacionada ao canal do crédito serviu de base para a realização do presente estudo, assim como a literatura referente ao canal de tomada de risco, haja vista a dificuldade de se encontrar trabalhos relativos, especificamente, a influência da política monetária sobre os seguros contratados pelos bancos e, por conseguinte, sobre o spread de crédito. Nesse sentido, o presente trabalho contribui com a literatura ao elaborar um estudo referente aos canais de transmissão do crédito (credit channel) e de tomada de risco (risk-taking channel). O estudo se diferencia dos trabalhos existentes ao destacar a influência da política monetária sobre o spread de crédito por meio dos seguros contratados pelo setor bancário.

Além desta Introdução, o trabalho encontra-se dividido da seguinte maneira. Na Seção 2 é apresentada uma breve resenha da literatura sobre mecanismos de transmissão de política monetária por meio dos canais do crédito e de tomada de risco. Na Seção 3 é elaborada a análise empírica. E por último, na Conclusão, são expostos os principais resultados obtidos pelo estudo.

\section{TRANSMISSÃO DA POLÍTICA MONETÁRIA PELO CANAL DO CRÉDITO E PELO CANAL DE TOMADA DE RISCO}

Existe considerável literatura sobre o papel que o crédito desempenha como mecanismo de transmissão da política monetária, utilizando dados de séries temporais agregados. Os trabalhos de Bernanke e Blinder (1992), Kashyap et alii (1993) e Gertler e Gilchrist (1993) representam importantes referências para esta literatura. Na década de 2000, podem ser mencionados, por exemplo, os trabalhos de Ehrmann et alii (2003) e Iacoviello e Minetti (2008).

Em relação ao mecanismo de transmissão de política monetária por meio do canal do crédito para o período que compreende o regime de metas para inflação no Brasil, os trabalhos elaborados por de Mello

\footnotetext{
${ }^{1}$ Apesar do canal de crédito ser tradicionalmente dividido em dois canais distintos, o canal de empréstimos bancários e o canal do balanço, o presente estudo centra-se no canal de empréstimos bancários. Para mais informações e descrições detalhadas em relação a estes dois canais, ver Bernanke e Gertler (1995).
} 
e Pisu (2010), Auel e Mendonça (2011) e Montes e Machado (2013) encontram importantes evidências que devem ser destacadas.

O trabalho de de Mello e Pisu (2010) analisa o canal de transmissão de política monetária pelos empréstimos bancários no Brasil usando dados agregados mensais de dezembro de 1995 a junho de 2008. A análise é realizada por meio de um modelo de correção de erro vetorial (VECM) que permite múltiplas relações de cointegrações entre as variáveis de interesse. O estudo encontra evidências de dois vetores de cointegração, que eles identificam como funções de demanda e oferta de empréstimos. A oferta de empréstimos é encontrada como sendo negativamente relacionada à taxa de certificado de depósito interbancário, sugerindo a existência de um canal de empréstimos para a transmissão monetária.

O trabalho de Auel e Mendonça (2011) analisa a relevância macroeconômica do canal do crédito no Brasil. Baseados em dados de 2002 a 2009, três conjuntos de modelos GMM são considerados: o primeiro conjunto analisou os efeitos dos choques sobre as variáveis econômicas que são essenciais para a oferta de crédito, o segundo conjunto considerou os efeitos das mesmas variáveis utilizadas no caso anterior sobre o spread de crédito, e o terceiro conjunto levou em conta os efeitos das mudanças nas condições do mercado de crédito sobre o produto. Os resultados apontam que os efeitos de choques econômicos sobre a oferta de crédito e sobre o spread de crédito estão de acordo com a teoria do canal do crédito. Além disso, é observado que choques sobre a taxa de juros não são transmitidos diretamente para a economia, mas por meio do canal do crédito.

O trabalho de Montes e Machado (2013) apresenta um modelo teórico e analisa empiricamente a transmissão da política monetária por meio do canal do crédito no Brasil. O estudo verifica se a política monetária, a atividade econômica e a maturidade do regime de metas para inflação afetam a oferta de crédito. As estimativas indicam que a oferta de crédito é estimulada quando a economia se aquece, quando a autoridade monetária reduz a taxa de juros e quando a credibilidade aumenta. As evidências também indicam que a atividade econômica e o emprego são afetados pela política monetária, e a oferta de crédito exerce influência sobre o emprego e o hiato do produto.

Por sua vez, outro tipo de mecanismo de transmissão da política monetária vem ganhando grande atenção e foi recentemente denominado como o canal de tomada de risco (risk-taking channel). Este canal diz respeito a como as mudanças nas taxas de juros de política monetária afetam tanto a percepção de risco como também a tolerância ao risco. Borio e Zhu (2008) ressaltam que os estudos relacionados aos mecanismos de transmissão não têm dado atenção suficiente para a ligação entre a política monetária e a percepção e apreçamento do risco - ou seja, ao canal de tomada de risco da política monetária. Nesse contexto, eles desenvolveram este conceito, o compararam com as visões atuais do mecanismo de transmissão, exploraram a sua ligação com a "liquidez" e analisaram a sua interação com funções de reação de política monetária. Argumentaram, por fim, que as mudanças no sistema financeiro e a regulamentação prudencial podem ter aumentado a importância do canal de tomada de risco e que paradigmas macroeconômicos vigentes e modelos associados não são adequados para capturá-lo, reduzindo assim a sua eficácia como guias para a política monetária.

Com base neste conceito de canal de tomada de risco, Altunbas et alii (2009) realizaram uma avaliação empírica para a zona do Euro e EUA, pela qual encontraram evidências de um canal de empréstimos bancários por meio do risco bancário. As evidências encontradas apontam que para avaliar a capacidade e vontade de um banco entregar novos empréstimos, as condições bancárias de risco precisam ser consideradas em conjunto com outros indicadores (isto é, tamanho, liquidez e capitalização), os quais são tradicionalmente utilizados na literatura sobre canal do crédito bancário. Os resultados sugerem que a manutenção das taxas de juros em patamares muito baixos durante um período prolongado de tempo causa um aumento na propensão a assumir riscos por parte dos bancos.

O estudo elaborado por Montes e Peixoto (2012) mostrou que, se por um lado a credibilidade é importante para a condução da política monetária, por outro lado, uma maior credibilidade pode, eventualmente, estimular a criação de bolhas no mercado de crédito e preços de ativos por meio do canal de tomada de risco. $\mathrm{O}$ trabalho buscou: 
(i) analisar o impacto das ações do banco central e do ambiente macroeconômico sobre a percepção de risco dos bancos, e

(ii) analisar a influência dessa percepção de risco dos bancos sobre o spread de crédito, considerando tanto o canal do crédito e o canal de tomada de risco.

Com base em uma análise econométrica, o trabalho forneceu evidências sobre:

(i) o "paradoxo de credibilidade" e o canal de tomada de riscos,

(ii) a influência da política monetária sobre a percepção de risco dos bancos e sobre o spread de crédito,

(iii) a natureza pró-cíclica dos bancos em relação à atividade econômica, e

(iv) a aderência do indicador anticíclico (chamado gap de crédito) proposto em Basileia III para o caso brasileiro.

Inequivocamente, a política monetária influencia a tomada de risco dos bancos (Brunnermeier, 2001, Rajan, 2005, Gambacorta, 2009, Altunbas et alii, 2009). Alterações na taxa básica de juros, como também nas reservas compulsórias, afetam o custo do capital, influenciando, por conseguinte, a atividade econômica. Como sugere o trabalho de Carling et alii (2007), é esperado que um(a) aumento (redução) da taxa de juros nominal impacte negativamente (positivamente) a atividade econômica e aumente (reduza) a taxa de inadimplência. Por sua vez, uma taxa de inadimplência alta é um indicativo do elevado risco de crédito ao qual estão expostos os bancos, e que caso não seja administrado corretamente pode levar a crises no sistema bancário. O aumento da inadimplência é capaz também de gerar redução na lucratividade dos bancos e, por conseguinte, reduzir a atividade econômica devido à restrição na concessão de novos empréstimos. A alta da inadimplência é um risco, mas também um incentivo à contratação de seguro de crédito. Ou seja, o aumento da inadimplência estimula a demanda por seguro de crédito.

Acerca do canal de tomada de risco, não há estudos que abordem a influência da política monetária sobre a percepção de risco dos bancos medida pelos seguros de crédito contratados e, por conseguinte, pelo spread de crédito. Ou seja, não existe, para o caso brasileiro, qualquer estudo acerca de como esta percepção de risco afeta o montante de seguros de crédito contratado e o spread de taxa de juros cobrado nas operações de crédito.

\section{ANÁLISE EMPÍRICA}

A análise elaborada está baseada nos trabalhos de Auel e Mendonça (2011) e Montes e Peixoto (2012) acerca da transmissão da política monetária pelo canal do crédito e pelo canal de tomada de risco. Entretanto, o presente trabalho se diferencia dos demais trabalhos elaborados, pois destaca a influência da política monetária sobre a percepção de risco dos bancos, e como esta impacta os seguros contratados contra perdas com operações de crédito às pessoas físicas e, por conseguinte, como estes afetam o spread de crédito. Em outras palavras, nessa seção são fornecidas evidências empíricas para a economia brasileira do impacto exercido pela política monetária sobre o spread de crédito considerando os seguros contratados contra perdas com operações de crédito às pessoas físicas.

De acordo com a SUSEP, órgão fiscalizador do mercado de seguros nacional, o seguro de crédito é definido como uma modalidade de seguro que tem por objetivo ressarcir o segurado (credor), nas operações de crédito realizadas com clientes domiciliados no país, das perdas líquidas definitivas causadas por devedor insolvente. Suas principais coberturas são: Operações de Consórcio, Operações de Empréstimo Hipotecário e Operações de Arrendamento Mercantil ("Leasing”). ${ }^{2}$

\footnotetext{
${ }^{2}$ Este seguro é geralmente contratado por empresas que realizam operações de crédito em suas vendas - tanto para pessoa fisica como para pessoa jurídica - ou intermediários de operações de crédito, financiamento e investimento; consórcios, empresas de
} 
As principais características do seguro de crédito são:

a - Participação obrigatória do segurado, que visa a manter o interesse deste na seleção dos riscos, assim como no resultado das ações judiciais e extrajudiciais;

b - Globalidade das operações, que tem por objetivo evitar que a entidade de crédito somente inclua na apólice os riscos de maior vulto e probabilidade; e

c - Limite de crédito, que é o estabelecimento de um limite máximo de crédito, evitando assim as fraudes e o excesso de exposição do segurado.

Este tipo de seguro tem as seguintes modalidades:

a - Riscos Comerciais - tem por objetivo cobrir as operações de crédito realizadas pelo segurado, somente com pessoas jurídicas domiciliadas no país; e

b - Quebra de Garantia - tem por objetivo cobrir as operações de crédito realizadas pelo segurado, especialmente aquelas relativas à venda de bens de consumo, para pessoas físicas ou jurídicas domiciliadas no país.

A principal característica desta modalidade é a existência de garantias reais, em que os bens envolvidos na operação são utilizados como garantia ao segurado e à Seguradora.

É importante destacar que o presente estudo utiliza na análise dados acerca do montante total pago com seguros contratados pelas entidades financeiras, especialmente bancos, para cobertura do risco de crédito doméstico junto às pessoas físicas (extraídos do SES da SUSEP).

\subsection{Descrição da análise e metodologia}

A análise empírica elaborada por Auel e Mendonça (2011) aborda como choques nas variáveis macroeconômicas afetam o spread de crédito e, consequentemente, a produção industrial, identificando dessa maneira uma possibilidade de transmissão da política monetária no Brasil pelo canal do crédito. Por sua vez, o trabalho de Montes e Peixoto (2012) analisa o impacto das ações do banco central e do ambiente macroeconômico sobre a percepção de risco dos bancos, e a influência dessa percepção de risco dos bancos sobre o spread de crédito, considerando tanto o canal do crédito e o canal de tomada de risco.

A Figura 1 esquematiza a análise do presente estudo, o qual utilizou o prêmio de seguro de crédito como variável explicativa do spread de crédito. Ademais, com base nos trabalhos existentes na literatura sobre canal de crédito e na teoria econômica, foram incluídas outras variáveis macroeconômicas nas estimações.

A análise empírica considerou o método de mínimos quadrados ordinários (OLS) e o método de momentos generalizado (GMM). De acordo com Hansen (1982), uma das razões para utilizar o método GMM é que, enquanto o método OLS geralmente apresenta problemas de autocorrelação serial, heterocedasticidade e não linearidade, fatores comuns em séries temporais macroeconômicas, o GMM oferece estimadores consistentes para a regressão.

É importante notar que os coeficientes estimados por GMM são consistentes somente se as variáveis instrumentais utilizadas na análise são exógenas. Portanto, a hipótese de exogeneidade dos instrumentos requer que essas variáveis não afetem diretamente a variável dependente. Neste sentido, um procedimento padrão que garante tal hipótese baseia-se em Johnston (1984), ou seja, os instrumentos escolhidos foram datados do período $t-1$ ou anterior. Ainda em relação ao GMM, Cragg (1983)

factoring e outras, caracterizados desta forma como segurados das operações de crédito. Os segurados também são os responsáveis pelo pagamento do prêmio de seguro. Os contratantes da operação de crédito, ou seja, os devedores são denominados garantidos, e é sobre eles que incide o risco de inadimplência. 
Figura 1: Esquema de análise

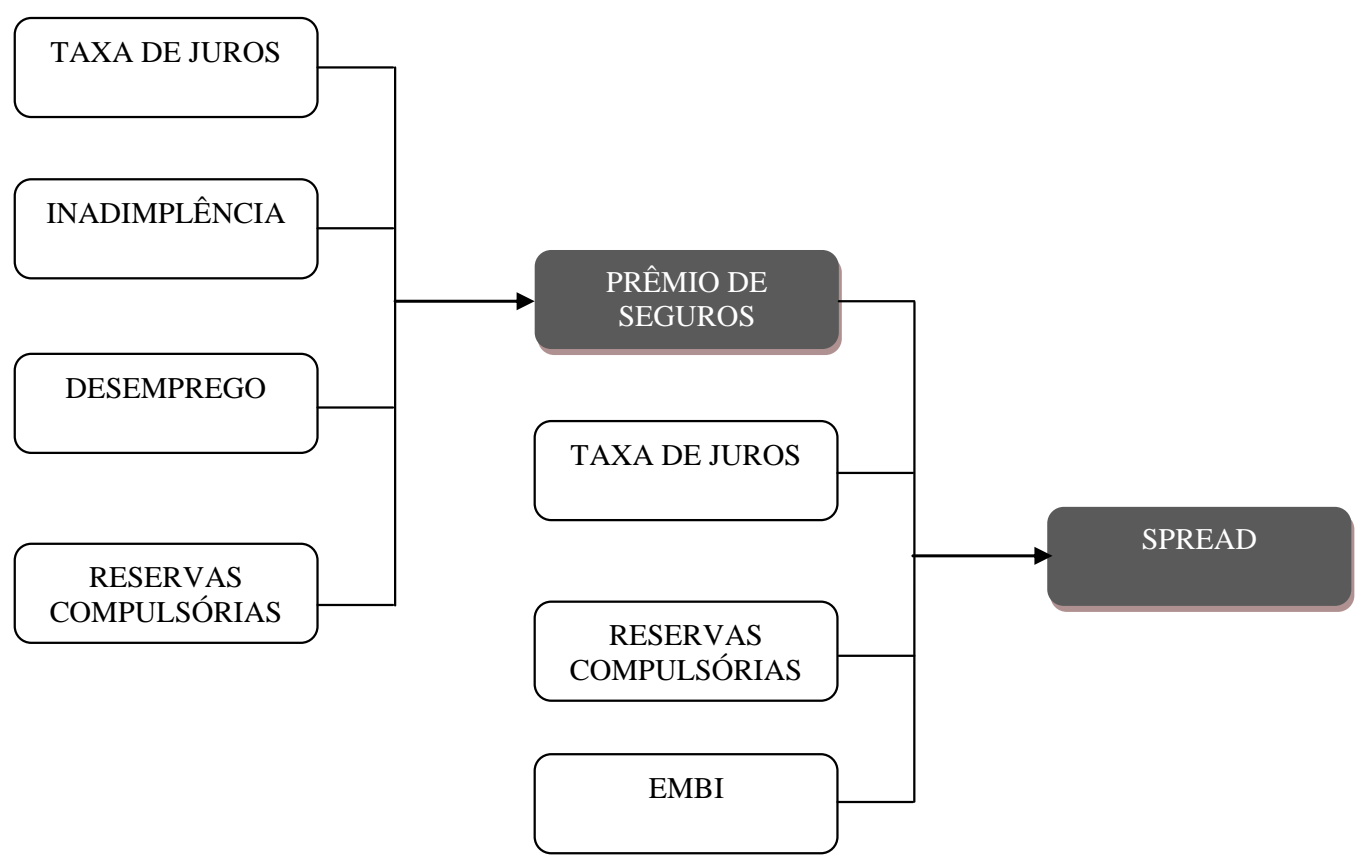

apontou que a análise de sobreidentificação tem um papel importante na seleção de variáveis instrumentais para melhorar a eficiência dos estimadores. Assim, realizou-se o teste $J$ padrão, com o objetivo de testar essa propriedade (Hansen, 1982).

\subsection{Dados}

O período analisado vai de janeiro de 2005 a maio de 2012. A escolha de iniciar a análise a partir de 2005 se justifica, pois se observa nesse período maior estabilidade do ambiente macroeconômico. As séries mensais utilizadas são as seguintes: ${ }^{3}$

Prêmio de Seguros (PREM) - Montante total pago pelas entidades financeiras, especialmente bancos, para cobertura do risco de crédito doméstico junto às pessoas físicas, já descontados os cancelamentos, restituições e descontos. Os dados utilizados foram extraídos do SES da SUSEP e contemplam o grupo de Crédito Doméstico - Risco Pessoa Física (Fonte: SES/SUSEP - Grupo_Ramo: 0870).

Spread (SPREAD) - Esta série representa o spread médio das operações de crédito com recursos livres referenciais para taxa de juros (pré-fixado) - Total pessoa físicas - em pontos percentuais. Referese ao diferencial entre as taxas de juros consolidadas das operações de crédito e os custos de

\footnotetext{
${ }^{3}$ Cabe esclarecer que para as variáveis expressas em valores monetários foi aplicado o logaritmo natural, e para aquelas expressas em níveis percentuais foi aplicado o seguinte ajuste, o qual também utiliza o logaritmo natural $(\ln (1+$ variavel $/ 100))$. Nesse sentido, a análise está baseada nas estimações das elasticidades.
} 
captação. O grupo pessoas físicas compreende cheque especial, crédito pessoal, aquisição de bens-veículos e outros bens (Fonte: série 3957/ BACEN - Departamento Econômico).

Risco país (EMBI) - O EMBI+ é um índice utilizado como variável proxy para o risco país. De um modo geral, o EMBI mostra a diferença entre a taxa de retorno dos títulos de países emergentes e a oferecida por títulos emitidos pelo Tesouro americano. Essa diferença é o spread, ou o spread soberano (Fonte: JP Morgan).

Taxa básica de juros - Selic (IR) - No Brasil, a taxa de juros (Selic) é o principal instrumento de política monetária no regime de metas de inflação. A série utilizada foi disponibilizada pelo BACEN e está expressa em pontos percentuais (Fonte: série 4189/ BACEN - Departamento de Operações do Mercado Aberto).

Taxa de inadimplência (INAD) - Esta variável representa a taxa de inadimplência do sistema financeiro privado nacional, ou seja, o nível de perda dos bancos em suas operações de crédito em pontos percentuais. Refere-se ao saldo das operações de empréstimo, financiamento, adiantamento e arrendamento mercantil, concedidas pelas instituições integrantes do sistema financeiro privado nacional, nas quais pessoas físicas e jurídicas residentes no país detém a maioria do capital votante. (Fonte: série 13673/ BACEN - Departamento Econômico).

Taxa de desemprego (U) - Relação entre o número de pessoas desocupadas (procurando trabalho) e o número de pessoas economicamente ativas num determinado período de referência. Expressa em pontos percentuais. (série: 10777/Fonte: IBGE).

Taxa de reservas compulsórias (RR) - Esta série está expressa em valores percentuais e representa um instrumento de política monetária que impacta diretamente o volume de crédito e, consequentemente, na disposição do sistema financeiro à tomada de risco. (Fonte: séries 1883, 1884, $1886 \mathrm{e}$ 17633/ BACEN - Departamento Econômico).

A primeira condição a ser testada antes de aplicar a análise econométrica é verificar se as séries são estacionárias. Portanto, foi aplicado o teste de raiz unitária KPSS (Kwiatkowski-Phillips-Schmidt-Shin) (ver Tabela A.1, no Apêndice). A vantagem deste teste resulta do baixo poder de testes convencionais, que tendem a não rejeitar a hipótese nula demasiadas vezes (Franses e Haldrup, 1994, Cati et alii, 1999). Ademais, Kwiatkowski et alii (1992) argumentam que o teste KPSS pode distinguir séries que aparentam ser estacionárias, séries que aparentam ter uma raiz unitária, e séries para as quais os dados não são suficientemente informativos para ter a certeza se são estacionárias ou integradas - como é o caso de séries com curta periodicidade. Os resultados mostram que todas as séries são $I(0)$.

A Figura 2 ilustra o comportamento das séries para o período analisado. É interessante destacar que para o período relativo à crise subprime houve fortes alterações nas séries de inadimplência, spread de crédito, reservas compulsórias, risco-país, prêmio de seguros de crédito e taxa básica de juros. 
Figura 2: Gráfico de comportamento das séries
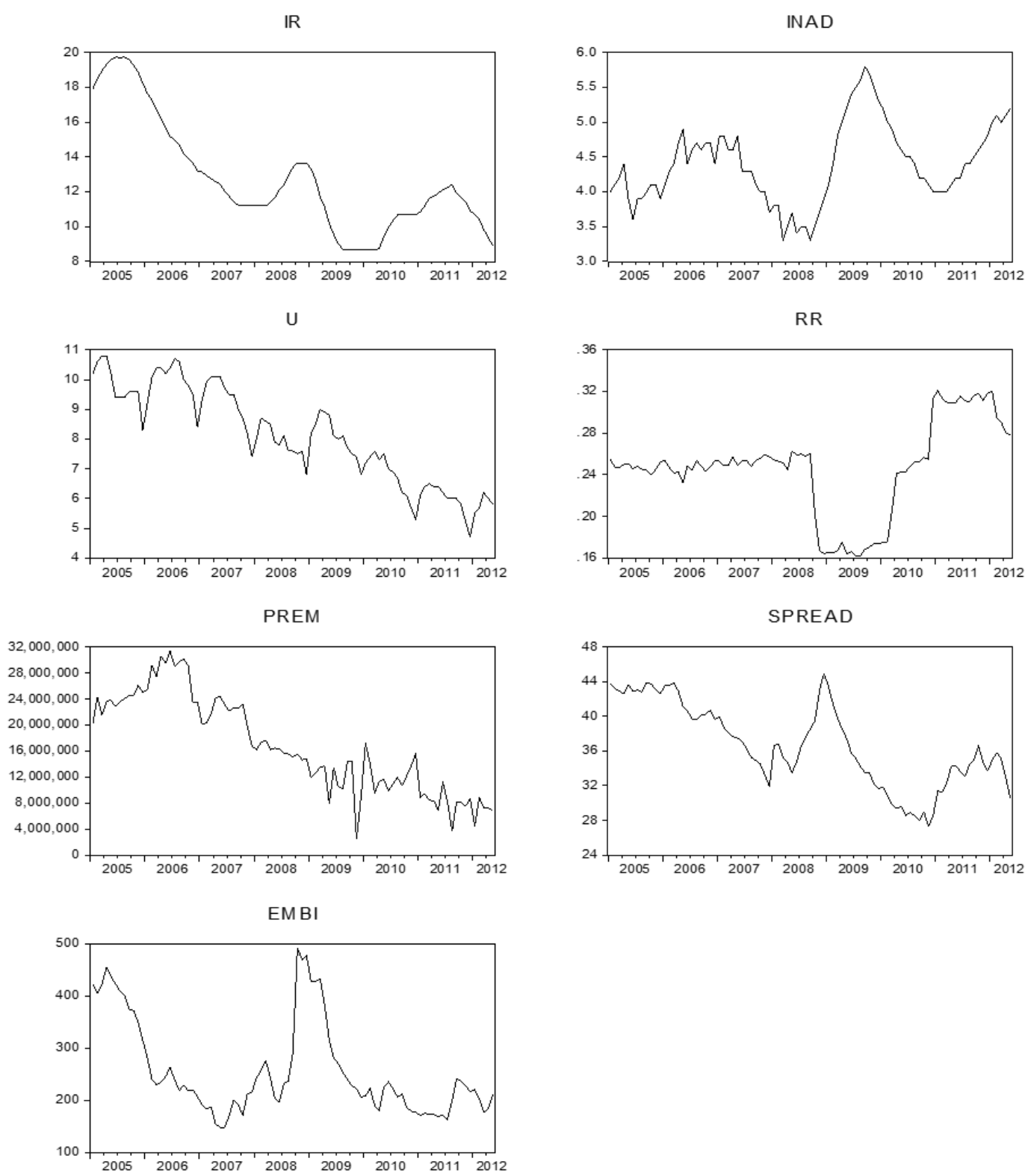


\subsection{Estimativas para a influência de variáveis macroeconômicas e políticas mo- netárias sobre o prêmio de seguro de crédito}

De forma a buscar evidências acerca da influência de variáveis econômicas e políticas monetárias sobre o prêmio de seguro de crédito para pessoa física, foi estimada a seguinte equação, cuja especificação é:

$$
P R E M_{t}=\beta_{0}+\beta_{1} P R E M_{t-1}+\beta_{2} I R_{t-1}+\beta_{3} I N A D_{t-7}+\beta_{4} U_{t-1}+\beta_{5} R R_{t-5}+\bar{\omega}_{t}
$$

em que $\bar{\omega}_{t}$ representa um termo de erro. Cabe esclarecer que a escolha dos lags foi determinada por meio da metodologia "geral para o específico", utilizando os critérios de informação tradicionalmente aplicados (Schwarz e Akaike), além do princípio da parcimônia e com base na teoria econômica.

A Tabela 1 apresenta os resultados das estimativas por OLS e GMM.

Sobre a estimativa por OLS, a estatística $\mathrm{F}$ indica que a regressão como um todo é significativa. Além disso, o resultado do teste RESET de Ramsey indica que a regressão não apresenta problema de especificação de modelo. Ademais, os testes para detectar heteroscedasticidade e autocorrelação indicam que os resíduos estimados não apresentam esses problemas. Em relação ao GMM, o resultado do teste- $J$ indicou que todo o modelo está corretamente especificado.

Considerando a influência das políticas monetárias sobre o prêmio de seguros de crédito, as estimativas apresentam significância estatística tanto para a taxa de juros Selic como também para a taxa de reservas compulsórias. Os coeficientes estimados para IR e RR confirmam as relações positivas que exercem sobre o prêmio de seguros de crédito. Assim, políticas monetárias contracionistas (expansionistas) induziriam os bancos a contratarem mais (menos) seguros. As estimativas mostram que limitando os recursos disponíveis para empréstimos, ou seja, aumentando o nível das reservas compulsórias, este instrumento de política monetária provocaria um aumento na procura por seguros de crédito. No entanto, somente foi verificada significância estatística por meio do método GMM.

O desemprego é uma das principais causas da inadimplência no país, respondendo por um terço dos casos (33\%) no mês de setembro de 2012 - como aponta pesquisa divulgada pela Boa Vista Serviços, administradora do SCPC (Serviço Central de Proteção ao Crédito). Portanto, quando o desemprego aumenta, haverá uma maior exposição dos bancos ao risco de crédito e, por conseguinte, estes buscarão maior proteção por meio do seguro de crédito. As estimativas mostram relações positivas entre desemprego e PREM e inadimplência e PREM. 
Tabela 1: Estimativas OLS e GMM (variável dependente: PREM)

\begin{tabular}{|c|c|c|}
\hline Variáveis Explicativas & OLS & GMM \\
\hline \multirow{3}{*}{ C } & $9.580^{* * *}$ & $7.091^{\text {*** }}$ \\
\hline & $(1.471)$ & $(0.788)$ \\
\hline & [6.508] & [8.993] \\
\hline \multirow{3}{*}{ PREM(-1) } & $0.262^{* *}$ & $0.440^{* * *}$ \\
\hline & $(0.104)$ & $(0.059)$ \\
\hline & {$[2.511]$} & [7.399] \\
\hline \multirow{3}{*}{$\operatorname{IR}(-1)$} & $4.586^{* *}$ & $4.439^{* * *}$ \\
\hline & $(2.000)$ & $(0.810)$ \\
\hline & [2.292] & {$[5.478]$} \\
\hline \multirow{3}{*}{$\operatorname{INAD}(-7)$} & $11.264^{*}$ & $11.474^{* * *}$ \\
\hline & $(6.747)$ & (1.885) \\
\hline & [1.669] & [6.086] \\
\hline \multirow{3}{*}{$\mathrm{U}(-1)$} & $18.728^{* * *}$ & $11.970^{* * *}$ \\
\hline & (3.984) & $(1.487)$ \\
\hline & {$[4.700]$} & {$[8.048]$} \\
\hline \multirow{3}{*}{$\mathrm{RR}(-5)$} & 0.545 & $0.997^{* * *}$ \\
\hline & $(0.873)$ & $(0.292)$ \\
\hline & {$[0.624]$} & {$[3.415]$} \\
\hline $\mathrm{R}^{2}$ & 0.743 & 0.690 \\
\hline $\mathrm{R}^{2}$ ajustado & 0.726 & 0.660 \\
\hline Jarque-Bera & 786.480 & \\
\hline Prob(Jarque-Bera) & 0.000 & \\
\hline Rank & & 30 \\
\hline Estatística F & 43.969 & \\
\hline $\operatorname{Prob}(\mathrm{F})$ & 0.000 & \\
\hline Estatística J & & 13.830 \\
\hline Prob(Estatística J) & & 0.950 \\
\hline Ramsey RESET (1) & 0.606 & \\
\hline Prob(Ramsey RESET) & 0.547 & \\
\hline Heterocedasticidade ARCH (1) & 0.005 & \\
\hline Prob(Heterocedasticidade) & 0.940 & \\
\hline Breusch-Godfrey Teste LM (1) & 0.326 & \\
\hline Prob(Breusch-Godfrey Teste LM) & 0.570 & \\
\hline Breusch-Godfrey Teste LM (2) & 2.083 & \\
\hline Prob(Breusch-Godfrey Teste LM) & 0.132 & \\
\hline Breusch-Godfrey Teste LM (3) & 1.584 & \\
\hline Prob(Breusch-Godfrey Teste LM) & 0.201 & \\
\hline Breusch-Godfrey Teste LM (4) & 1.297 & \\
\hline Prob(Breusch-Godfrey Teste LM) & 0.2780 & \\
\hline
\end{tabular}




\subsection{Estimativas para a influência das políticas monetárias e do prêmio de seguro de crédito sobre o spread de crédito}

De forma a buscar evidências acerca da influência que exercem as políticas monetárias e o prêmio de seguro de crédito para pessoa física sobre o spread de crédito, foi estimada a seguinte equação: ${ }^{4}$

$$
\begin{gathered}
S P R E A D_{t}=\gamma_{0}+\gamma_{1} S P R E A D_{t-1}+\gamma_{2} I T_{t-5}+ \\
\gamma_{3} P_{R E M_{t-4}}+\gamma_{4} R R_{t-3}+\gamma_{5} E M B I_{t-1} * D+\lambda_{t}
\end{gathered}
$$

em que $\lambda_{t}$ representa um termo de erro. Novamente, cabe ressaltar que a escolha dos lags foi determinada por meio da metodologia "geral para o específico", utilizando os critérios de informação tradicionalmente aplicados (Schwarz e Akaike), além do princípio da parcimônia, e com base na teoria econômica.

A Tabela 2 apresenta os resultados das estimativas por OLS e GMM.

Em relação à estimativa por OLS, a estatística $\mathrm{F}$ indica que a regressão como um todo é significativa. Além disso, o resultado do teste RESET de Ramsey indica que a regressão não apresenta problema de especificação de modelo. Ademais, os testes para detectar heteroscedasticidade e autocorrelação indicam que os resíduos estimados não apresentam esses problemas. No tocante ao GMM, o resultado do teste- $J$ indicou que o modelo está corretamente especificado.

Conforme verificado por Auel e Mendonça (2011) e Montes e Peixoto (2012), nos estudos sobre a influência das políticas monetárias sobre o spread de crédito, os coeficientes estimados para as variáveis IR e RR foram positivos e estatisticamente significativos. Tal evidência, conforme apresentado por Montes e Peixoto (2012), indica que a variável SPREAD é um bom indicador para a manifestação do canal de crédito no regime de metas de inflação no Brasil.

Assim como encontrado por Auel e Mendonça (2011), o risco país (EMBI) mostrou-se significativo e positivamente relacionado com o SPREAD. Portanto, no que o risco-país se eleva e, assim, aumenta a probabilidade de default, este evento deve repercutir diretamente na magnitude do spread bancário, pois o ambiente de incerteza gerado contamina as percepções dos agentes que operam no sistema financeiro.

No tocante a influência do prêmio de seguro (PREM) sobre o SPREAD, as evidências apontam para uma relação positiva entre este e o SPREAD. Os resultados sugerem que o spread de crédito é sensível ao montante de seguros pagos pelas entidades financeiras, especialmente bancos, para cobertura do risco de crédito doméstico junto às pessoas físicas. Ou seja, no que esse montante aumenta isso indica que as entidades financeiras percebem um aumento no risco de crédito, e, portanto um aumento da inadimplência, e assim reagem aumentando o spread de crédito. Essa percepção, por sua vez, pode ser resultado da adoção de políticas monetárias contracionistas por parte do banco central.

\footnotetext{
${ }^{4} \mathrm{~A}$ fim de capturar os choques financeiros internacionais sobre a economia brasileira da crise das hipotecas subprime, uma variável DUMMY $(D)$ foi incluída. A DUMMY assume o valor 1 para o período de 2008.08 para 2008.12 e zero caso contrário. Esta data de início foi escolhida porque imediatamente após 2008.08, ocorreu a quebra do banco Lehman Brothers. Espera-se que os coeficientes desta variável apresentem sinais positivos, porque, em ambientes turbulentos, gerados por crises, criam incertezas que induzem os bancos a aumentar suas disposições e, portanto, restringir o crédito, como, por exemplo, aumentando o spread de crédito.
} 
Tabela 2: Estimativas OLS e GMM (variável dependente: SPREAD)

\begin{tabular}{|c|c|c|}
\hline Variáveis Explicativas & OLS & GMM \\
\hline \multirow{3}{*}{ C } & -0.036 & -0.020 \\
\hline & $(0.035)$ & $(0.028)$ \\
\hline & {$[-1.016]$} & {$[-0.709]$} \\
\hline \multirow{3}{*}{ SPREAD(-1) } & $0.759^{* * *}$ & $0.701^{* * *}$ \\
\hline & $(0.057)$ & $(0.077)$ \\
\hline & {$[13.220]$} & {$[9.061]$} \\
\hline \multirow{3}{*}{$\operatorname{IR}(-5)$} & $0.1966^{* * *}$ & $0.263^{* * *}$ \\
\hline & $(0.073)$ & $(0.078)$ \\
\hline & {$[2.686]$} & [3.377] \\
\hline \multirow{3}{*}{ PREM(-4) } & $0.004^{* *}$ & $0.004^{*}$ \\
\hline & $(0.002)$ & $(0.001)$ \\
\hline & [2.141] & {$[2.286]$} \\
\hline \multirow{3}{*}{$\mathrm{RR}(-3)$} & $0.036^{*}$ & $0.024^{* *}$ \\
\hline & $(0.019)$ & $(0.011)$ \\
\hline & {$[1.852]$} & {$[2.106]$} \\
\hline \multirow{3}{*}{$\operatorname{EMBI}(-1)^{*} \mathrm{D}$} & $0.003^{* * *}$ & $0.005^{* * *}$ \\
\hline & $(0.001)$ & $(0.001)$ \\
\hline & {$[5.076]$} & [3.623] \\
\hline $\mathrm{R}^{2}$ & 0.954 & 0.945 \\
\hline $\mathrm{R}^{2}$ ajustado & 0.951 & 0.942 \\
\hline Jarque-Bera & 25.688 & \\
\hline Prob(Jarque-Bera) & 0.000 & \\
\hline Rank & & 19 \\
\hline Estatística F & 325.050 & \\
\hline $\operatorname{Prob}(\mathrm{F})$ & 0.000 & \\
\hline Estatística J & & 7.492 \\
\hline Prob(Estatística J) & & 0.875 \\
\hline Ramsey RESET (1) & 0.160 & \\
\hline Prob(Ramsey RESET) & 0.689 & \\
\hline Heterocedasticidade ARCH (1) & 1.141 & \\
\hline Prob(Heterocedasticidade) & 0.288 & \\
\hline Breusch-Godfrey Teste LM (1) & 0.867 & \\
\hline Prob(Breusch-Godfrey Teste LM) & 0.354 & \\
\hline Breusch-Godfrey Teste LM (2) & 2.669 & \\
\hline Prob(Breusch-Godfrey Teste LM) & 0.075 & \\
\hline Breusch-Godfrey Teste LM (3) & 2.090 & \\
\hline Prob(Breusch-Godfrey Teste LM) & 0.108 & \\
\hline Breusch-Godfrey Teste LM (4) & 1.834 & \\
\hline Prob(Breusch-Godfrey Teste LM) & 0.131 & \\
\hline
\end{tabular}




\subsection{Sistema de equações simultâneas para o Prêmio de Seguros e o Spread}

A análise anterior indica que políticas monetárias, por meio de reservas compulsórias e da taxa de juros, influenciam o volume de seguros contatados e aumentam o spread de crédito, reforçando a ideia de que o canal de tomada de riscos e o canal de crédito estariam operando no Brasil.

Uma maneira de dar robustez às estimações individuais realizadas por OLS e GMM e, assim, observar os canais de transmissão de tomada de riscos e do crédito é a estimativa utilizando sistema de equações simultâneas. Para o tratamento de possíveis problemas de endogeneidade a estimação do sistema foi feita por GMM. O sistema de equações estimado é:

Sistema $\left\{\begin{array}{c}\operatorname{PREM}_{t}=\alpha_{0}+\alpha_{1} \mathrm{PREM}_{t-1}+\alpha_{2} I R_{t-1}+\alpha_{3} I N A D_{t-7}+\alpha_{4} U_{t-1}+\alpha_{5} R R_{t-5}+\xi_{t} \\ \text { SPREADt }=\phi_{0}+\phi_{1} S P R E A D_{t-1}+\phi_{2} I R_{t-5}+\phi_{3} P R E M_{t-4}+\phi_{4} R R_{t-3} \\ +\phi_{5} E M B I_{t-1} * D+\tau_{t}\end{array}\right.$

em que, $\xi_{t}$ e $\tau_{t}$ são termos de erro aleatório. A Tabela 3 mostra as estimativas do sistema.

Todos os coeficientes das variáveis analisadas têm sinais de acordo com a visão teórica e com significância estatística. Vale ressaltar que os coeficientes obtidos pelo sistema são mais precisos do que os estimados nas especificações individuais - como se observa, os erros-padrão são menores.

Tabela 3: Sistema GMM (PREM e SPREAD)

\begin{tabular}{|c|c|c|c|}
\hline \multicolumn{4}{|c|}{ SISTEMA GMM - PREM E SPREAD } \\
\hline Variáveis Dependentes & PREM & SPREAD & \\
\hline \multirow[t]{2}{*}{ Variáveis Explicativas } & \multicolumn{3}{|c|}{ Variáveis Explicativas } \\
\hline & $7.156^{* * *}$ & $\mathrm{C}$ & -0.013 \\
\hline \multirow[t]{3}{*}{ C } & $(0.654)$ & & $(0,019)$ \\
\hline & [10.933] & & {$[-0.694]$} \\
\hline & $0.435^{* * *}$ & SPREAD(-1) & $0.668^{* * *}$ \\
\hline \multirow[t]{3}{*}{$\operatorname{PREM}(-1)$} & $(0,048)$ & & $(0.044)$ \\
\hline & [8.914] & & [15.184] \\
\hline & $4.609^{* * *}$ & $\operatorname{IR}(-5)$ & $0.296^{* * *}$ \\
\hline \multirow[t]{3}{*}{$\operatorname{IR}(-1)$} & $(0.682)$ & & $(0.046)$ \\
\hline & [6.752] & & [6.339] \\
\hline & $11.719^{* * *}$ & PREM(-4) & $0.004^{* * *}$ \\
\hline \multirow[t]{3}{*}{$\operatorname{INAD}(-7)$} & $(1.492)$ & & $(0.001)$ \\
\hline & {$[7.854]$} & & [3.195] \\
\hline & $11.695^{* * *}$ & $\mathrm{RR}(-3)$ & $0.026^{* * *}$ \\
\hline \multirow[t]{3}{*}{$\mathrm{U}(-1)$} & $(1.163)$ & & $(0.008)$ \\
\hline & {$[10.050]$} & & {$[3.057]$} \\
\hline & $0.990^{* * *}$ & $\operatorname{EMBI}(-1)^{*} \mathrm{D}$ & $0.005^{* * *}$ \\
\hline \multirow[t]{2}{*}{$\mathrm{RR}(-5)$} & $(0.248)$ & & $(0.001)$ \\
\hline & [3.977] & & [6.941] \\
\hline $\mathrm{R}^{2}$ & 0.688 & & 0.943 \\
\hline $\mathrm{R}^{2}$ ajustado & 0.664 & & 0.939 \\
\hline Estatística J & & 0.228 & \\
\hline Prob(Estatística J) & & 0.99 & \\
\hline
\end{tabular}


Os resultados apontam que reduções (elevações) na taxa de juros ou de reservas compulsórias provocam reduções (elevações) na contratação de seguros de crédito, e consequentemente reduziriam (aumentariam) os riscos observados no mercado de crédito.

Vale observar que as políticas monetárias afetam diretamente o spread de crédito, suscitando assim a existência do canal de crédito. Por sua vez, é importante destacar também que, as políticas monetárias afetam indiretamente o spread praticado nas operações de crédito por meio da percepção de risco dos bancos, a qual se reflete sobre o montante contratado de seguros de crédito, sugerindo assim, a existência de um canal de tomada de risco que acaba por influenciar as operações de crédito na economia.

Por fim, é importante ressaltar que uma redução (um aumento) na contratação de seguros de crédito resulta de uma melhora (piora) na percepção dos bancos em relação ao risco de crédito, o que possibilita ao banco assumir mais (menos) riscos e, portanto, aumentar (reduzir) sua exposição, por exemplo, reduzindo (aumentando) o spread nas operações de crédito.

\section{CONCLUSÃO}

Este estudo analisa a influência da política monetária sobre o processo de contratação de seguro referente às perdas com operações de concessão de crédito bancário às pessoas físicas (Seguro de Crédito - Pessoa Física) e investiga o impacto exercido pela contratação desta modalidade de seguro sobre o spread de crédito, e, portanto, sobre o risco assumido por bancos com essas operações.

Visto a carência de trabalhos que relacionam o seguro ao mecanismo de transmissão da política monetária, o presente estudo contribui com a literatura ao elaborar um estudo referente aos canais de transmissão do crédito (credit channel) e de tomada de risco (risk-taking channel) usando o seguro referente às perdas com operações de concessão de crédito bancário às pessoas físicas. A literatura de canais de transmissão do crédito e de tomada de risco serviu de alicerce ao presente trabalho, que se diferencia dos demais trabalhos elaborados ao destacar a influência da política monetária sobre a percepção de risco dos bancos, e como esta impacta os seguros contratados contra perdas com operações de crédito às pessoas fisicas e, por conseguinte, como estes afetam o spread de crédito.

A análise empírica elaborada está baseada nos trabalhos de Auel e Mendonça (2011) e Montes e Peixoto (2012) acerca da transmissão da política monetária pelo canal do crédito e pelo canal de tomada de risco. Esta pesquisa utilizou o prêmio de seguro de crédito como variável explicativa do spread de crédito. Ademais, com base nos trabalhos existentes na literatura sobre canal de crédito e na teoria econômica, foram incluídas outras variáveis macroeconômicas nas estimações. A amostra de dados utilizados varia de janeiro de 2005 até maio de 2012 .

Os resultados indicam que há influência de variáveis macroeconômicas e de política monetária sobre o prêmio de seguro de crédito. As estimativas apresentam significância estatística tanto para a taxa de juros Selic como também para a taxa de reservas compulsórias. Os coeficientes estimados para estas variáveis confirmam as relações positivas sobre o prêmio de seguros de crédito. Assim, políticas monetárias contracionistas (expansionistas) induziriam os bancos a contratarem mais (menos) seguros. As estimativas mostram que limitando os recursos disponíveis para empréstimos, ou seja, aumentando o nível das reservas compulsórias, este instrumento de política monetária provocaria um aumento na procura por seguros de crédito. As estimativas mostram relações positivas entre desemprego e prêmio de seguros e inadimplência e premio de seguros.

As estimativas para a influência da política monetária e do prêmio de seguro de crédito sobre o spread de crédito estão em linha com os estudos de Auel e Mendonça (2011) e Montes e Peixoto (2012). Os coeficientes estimados para as variáveis taxa básica de juros e taxas de reservas compulsórias foram positivos e estatisticamente significativos. Tal evidência, conforme apresentado por Montes e Peixoto (2012), indica que a variável spread é um bom indicador para a manifestação do canal de crédito no regime de metas de inflação no Brasil. O risco país (EMBI) mostrou-se significativo e positivamente relacionado com o spread, assim como em Auel e Mendonça (2011). As evidências apontam para uma 
relação positiva entre o prêmio de seguro e o spread. Os resultados sugerem que o spread de crédito é sensível ao montante de seguros pagos pelas entidades financeiras, especialmente bancos, para cobertura do risco de crédito doméstico junto às pessoas físicas.

Finalmente, aplica-se um sistema de equações simultâneas para o prêmio de seguros e o spread com objetivo de dar robustez às estimações individuais. Os coeficientes estimados são positivos e significativos, apontando que reduções (elevações) na taxa de juros ou de reservas compulsórias tenderiam a provocar reduções (elevações) na contratação de seguros de crédito. Por outro lado, é importante ressaltar que uma redução (um aumento) na contratação de seguros de crédito resultaria em melhora (piora) na percepção dos bancos em relação ao risco de crédito, o que possibilitaria ao banco assumir mais (menos) riscos e, portanto, aumentar (reduzir) sua exposição, por exemplo, reduzindo (aumentando) o spread nas operações de crédito.

\section{BIBLIOGRAFIA}

Altunbas, Y., Gambacorta, L., \& Marqués-Ibañez, D. (2009). An empirical assessment of the risk-taking channel. In BIS/ECB Conference on "Monetary policy and financial stability".

Auel, M. C. \& Mendonça, H. F. (2011). Macroeconomic relevance of credit channels: Evidence from an emerging economy under inflation targeting. Economic Modelling, 28:965-979.

Bernanke, B. \& Blinder, A. (1992). The federal funds rate and the channels of monetary transmission. The American Economic Review, 82:901-921.

Bernanke, B. \& Gertler, M. (1995). Inside the black box: The credit channel of monetary policy transmission. Journal of Economic Perspectives, 9:27-48.

Borio, C. \& Zhu, H. (2008). Capital regulation, risk-taking and monetary policy: A missing link in the transmission mechanism? Working Paper 268, Bank for International Settlements.

Brunnermeier, M. K. (2001). Asset Pricing under Asymmetric Information - Bubbles, Crashes, Technical Analysis and Herding. Oxford University Press, Oxford.

Carling, K., Jacobson, T., Linde, J., \& Roszbach, K. (2007). Corporate credit risk modeling and the macroeconomy. Journal of Banking and Finance, 31:845-868.

Cati, R. C., Garcia, M. G. P., \& Perron, P. (1999). Unit roots in the presence of abrupt governmental interventions with an application to Brazilian data. Journal of Applied Econometrics, 14:27-56.

Cragg, J. G. (1983). More efficient estimation in the presence of heteroscedasticity of unknown form. Econometrica, 51:751-763.

de Mello, L. \& Pisu, M. (2010). The bank lending channel of monetary transmission in Brazil: A VECM approach. The Quarterly Review of Economics and Finance, 50:50-60.

Ehrmann, M., Gambacorta, L., Martinez-Pages, J., Sevestre, P., \& Worms, A. (2003). Financial systems and the role of banks in monetary policy transmission in the Euro area. In Monetary Policy Transmission in the Euro Area: A Study by the Eurosystem Monetary Transmission Network. Cambridge University Press, Cambridge.

Franses, P. H. \& Haldrup, N. (1994). The effects of additive outliers on tests for unit roots and cointegration. Journal of Business and Economic Statistics, 12:471-478.

Gambacorta, L. (2009). Monetary policy and the risk-taking channel. BIS Quarterly Review, December. 
Gertler, M. \& Gilchrist, S. (1993). The role of credit market imperfections in the monetary transmission mechanism: Arguments and evidence. Scandinavian Journal of Economics, 95:43-64.

Hansen, L. P. (1982). Large sample properties of generalized method of moments estimators. Econometrica, 50:1029-1054.

Iacoviello, M. \& Minetti, R. (2008). The credit channel of monetary policy: Evidence from the housing market. Journal of Macroeconomics, 30:69-96.

Johnston, J. (1984). Econometric Methods. McGraw-Hill Book Co, Singapore, $3^{\text {rd }}$ edition.

Kashyap, A., Stein, J., \& Wilcox, D. (1993). Monetary policy and credit conditions: Evidence from the composition of external finance. The American Economic Review, 83:79-98.

Kwiatkowski, D., Phillips, P. C. B., Schmidt, P., \& Shin, Y. (1992). Testing the null hypothesis of stationarity against the alternative of a unit root. Journal of Econometrics, 54:159-178.

Mishkin, F. S. (1995). Symposium on the monetary transmission mechanism. Journal of Economic Perspectives, 9:3-10.

Montes, G. C. (2013). Credibility and monetary transmission channels under inflation targeting: An econometric analysis from a developing country. Economic Modelling, 30:670-684.

Montes, G. C. \& Machado, C. C. (2013). Credibility and the credit channel transmission of monetary policy: Theoretical model and econometric analysis for Brazil. Journal of Economic Studies, 40.

Montes, G. C. \& Peixoto, G. B. (2012). Risk-taking channel, bank lending channel and the "paradox of credibility": Evidence for Brazil. In ANPEC, editor, Encontro Nacional de Economia.

Rajan, R. G. (2005). Has financial development made the world riskier? Working Paper 11728, National Bureau of Economic Research. 


\section{A. APÊNDICE}

Tabela A.1 - Teste KPSS

\begin{tabular}{l|ccccc}
\hline \multirow{2}{*}{ Séries } & \multicolumn{5}{c}{ KPSS } \\
\cline { 2 - 6 } & Bandwidth & Estatística de Teste & Valor Crítico a 1\% & Valor Crítico a 5\% & Valor Crítico a 10\% \\
\hline PREM & 4 & 0.132 & 0.216 & 0.146 & 0.119 \\
IR & 7 & 0.205 & 0.216 & 0.146 & 0.119 \\
INAN & 7 & 0.056 & 0.216 & 0.146 & 0.119 \\
U & 5 & 0.082 & 0.216 & 0.146 & 0.119 \\
RR & 7 & 0.186 & 0.216 & 0.146 & 0.119 \\
SPREAD & 7 & 0.083 & 0.216 & 0.146 & 0.119 \\
EMBI & 6 & 0.103 & 0.216 & 0.146 & 0.119 \\
\hline
\end{tabular}

Nota: A escolha final pelo uso de intercepto ou intercepto e tendência foi feita baseada no critério de Schwarz.

A defasagem é o lag truncation escolhido para o Barlettkemel. Todos os testes utilizaram intercepto e tendência. 